\title{
Anomalous electrical conductivity and percolation in carbon nanotube composites
}

\author{
Chunsheng $\mathrm{Lu},{ }^{1, *}$ and Yiu-Wing $\mathrm{Mai}^{2}$ \\ ${ }^{1}$ Department of Mechanical Engineering, Curtin University of Technology, Perth, WA 6845, Australia \\ ${ }^{2}$ Centre for Advanced Materials Technology (CAMT), School of Aerospace, Mechanical and \\ Mechatronic Engineering J07, The University of Sydney, Sydney, NSW 2006, Australia
}

(October 18, 2007)

Transport properties of disordered multiphase materials, such as electrical and thermal conductivities, have been an active research area in statistical physics for decades. In a composite consisting of conductive fillers dispersed in an insulating matrix, there is a welldefined insulator-conductor transition when an infinite conductive network or path throughout the matrix is formed. This process can be well described by percolation theory $[1,2]$. Recently, carbon nanotube (CNT) reinforced composites and suspensions have attracted a great deal of attention due to their excellent properties and many potential applications. CNTs have a unique set of mechanical and physical properties, including extremely high Young's modulus, strength, electrical and thermal conductivities. Recent experiments show that CNTreinforced composites exhibit an electrical percolation with addition of 0.1 vol. $\%$ or less fillers, at which electrical conductivity rises sharply by several orders of magnitude [3-7]. Here, the percolation threshold of CNTs is closely dependent on their geometric factors (e.g. volume fraction, size, shape and orientation) and the interaction between them. It is a critical

\footnotetext{
* Corresponding author. e-mail: C.Lu@curtin.edu.au
} 
issue in producing conductive composites for use in films, coatings, and paints since the lower percolation threshold can reduce the loading of expensive CNTs, leading to lighter composites. In comparison with composites reinforced with isotropic particles, however, the percolation threshold of composites containing highly anisotropic conductor fillers such as CNTs is still not well understood.

The term "percolation" refers to the onset of a sharp transition or an infinite network (or cluster) at which long-range connectivity suddenly appears [1]. The electrical conductivity near the percolation threshold is anomalously greater than that predicted by traditional theoretical models, such as Maxwell, Hamilton-Crosser models etc [8]. Intuitively, the electrical percolation process in CNT composites is similar to traditional ones with the addition of isotropic conductive particles, but with an ultra-low percolation threshold. As shown in Fig. 1, near the percolation threshold, the probability or fraction of a CNT, $P$, on the infinite cluster obeys a power law and can be described by

$$
P \sim\left[\phi-\phi_{c}(\alpha)\right]^{\beta(\alpha)}
$$

where $\phi$ is volume fraction of CNT fillers (in vol. $\%$ or wt. $\%$ ), $\phi_{c}$ is percolation threshold, and $\beta$ is connectivity exponent. Similarly, the electrical conductivity, $\sigma$, in the system increases monotonically for $\phi>\phi_{c}$ but $\left(\phi-\phi_{c}\right)<<1$ and follows a universal power law

$\sigma \sim\left[\phi-\phi_{c}(\alpha)\right]^{t(\alpha)}$

where $t$ is conductivity exponent. Here, both the percolation threshold and connectivity (and conductivity) exponents are functions of aspect ratios of CNTs, $\alpha=L / D$, with $L$ length and $D$ diameter, respectively. It is well-known that the transport behavior of the system near the percolation threshold is controlled by the ubiquitous critical exponents, such as $\beta$ and $t$ in Eqs. (1) and (2). According to the percolation theory, $\beta=0.4$ and $t=2$ in a three-dimensional percolation (with an aspect ratio, $\alpha=1$ ) [2]. Thus, it is obvious that there is a striking 
difference between the ways that the connectivity, $P$, and the conductivity, $\sigma$, take off near $\phi$ $>\phi_{c}$. Just above the percolation threshold, the connectivity rises very steeply since $d P / d \phi$ becomes arbitrarily large as $\left(\phi-\phi_{c}\right) \ll 1$, the conductivity shows, however, a very soft rise since $d \sigma d \phi$ approaches zero as $\left(\phi-\phi_{c}\right)$ goes to zero. The physical reason for the contrast is that dead ends attached to the percolation cluster contribute to the fraction of connectivity but not to the electrical current it carries $[1,2]$.

Generally, there are only few cases where exact solutions on their percolation thresholds and exponents can be acquired. In most real situations, we have to resort to experiments or Monte Carlo simulations to obtain these values. However, as a typical critical phenomenon, renormalization, a powerful method for modeling a large heterogeneous system, can be also used in the study of percolation. Several models based on this concept have been successfully applied to similar behaviors in nanomaterials such as barrier properties of polymer-clay nanocomposites $[9,10]$.

The basic hypothesis of renormalization is the probability $p$ that a cell acts as a conductor is the same at all orders. Here, the essential step is the construction of a renormalization transformation, $p^{\prime}=R_{b}(p)$, between the original probability $p$ and the renormalized probability $p^{\prime}$ when the degree of coarse-graining $b$ in observation is changed [1, 11]. As a first approximation, let us consider a $b^{d}$-site Kadanoff cell with a ratio of coarse-graining $b=2$ and dimension $d=2$. To specify whether a cell is a conductor or not, the simplest way is to find if there is a conductive path through the cell. For the sake of simplicity, an element in a cell is considered as a conductor when it contains one or more CNTs. As shown in Fig. 2, a Kadanoff cell with four elements can have zero to four conductive elements; and hence there are totally $2^{4}=16$ possible combinations. Excluding multiplicities, there are 7 topologically different configurations. In a general case without specially indicated conductive directions, 
configurations 3 and 4 behave as conductive cells, which are highlighted in bold letters beneath the relevant configurations (see Fig. 2). For example, the conductive probability of configuration 3 is given by $4 p^{3}(1-p)$, where 4 is the number of possible combinations. Note configuration $2 \mathrm{c}$ is not considered as a conductive cell since its triangular distance is $\sqrt{ } 2$ times longer than the length of CNTs. By considering all the possible configurations, the renormalization transformation, $R_{2}(p)$, i.e., the probability $p^{\prime}$ that a cell of order $(i+1)$ is a conductor versus the probability $p$ that a cell of order $i$ or an element of order $(i+1)$ is a conductor, can be expressed as

$p^{\prime}=R_{2}(p)=p^{4}+4 p^{3}(1-p)$

This iterative equation crosses the straight line, $p^{\prime}=p$, at $p^{\prime}=p=p_{c}=0.768$, which is an unstable fixed point that separates a stable region from an unstable region. If $p<p_{c}$, the solution iterates to one stable point, $p=0$, as $i$ increases, and no electrical currency occurs. On the contrary, if $p>p_{c}$, the solution iterates to the other stable point, $p=1$, as $i$ increases, and the whole system acts as a conductor. Bifurcation of the solution occurs at $p=p_{c}$, which corresponds to the critical value of CNT content for the electrical percolation.

In thin films or coatings with the uniform distribution of CNTs, the corresponding volume fraction of a CNT in an element can be approximately estimated by the ratio of their volumes, that is, $\left(\pi D^{2} L / 4\right) /\left(L^{2} D\right)=(\pi / 4) D / L$. Thus, at the critical point, the percolation threshold of CNT content $\phi_{c}$ is obtained by

$\phi_{c}=\frac{\pi}{4} \frac{D}{L} p_{c}=\frac{0.60}{\alpha}$

where $p_{c}=0.768$. It is surprising to see that Eq. (4) is well consistent with that obtained by more complicated theoretical analyses and calculations $[12,13]$. It is necessary to mention that, however, to accurately model the conductivity of CNT composites, detailed knowledge 
about the geometric factors of CNTs such as size and shape are required, especially in the case of polydisperse distribution.

In addition to the pivotal influence of high aspect ratios, the strong interaction between rod-like CNTs could further reduce the percolation threshold. For example, the ionic surfactant, sodium dodecylsulfate, was used to disperse single-walled CNT bundles in water, and induced a depleted attraction between CNTs. The strength of the attraction is directly proportional to the micelle concentration and thus to the efficiency of electrical conductivity in CNT composites [5]. In other words, the interaction between CNTs can increase the conductive probability of a cell and further decrease the percolation threshold. For instance, configuration $2 \mathrm{c}$ in Fig. 2 could be transferred into a conductive cell due to the interaction between CNTs. However, quantitative understanding of the influence of the interaction potential between CNTs on the percolation threshold is still a big challenge.

In contrast to electrical conductivity, there seems to be no signature of the percolation threshold in thermal transport measurements on CNT composites although these two transport processes are described by the same Laplace equation [4]. A theoretical analysis based on finite element calculations was conducted to show the underlying reasons for the surprising and puzzling distinction between electrical and thermal transport behaviors in high aspect ratio fiber composites. However, in our opinion, the assertion on the lack of thermal percolation in CNT composites is worthy of further critical evaluation. Recent theoretical and computational studies have shown that conductivity exponents are in the range of $1.2-1.6$, which is in good agreement with experimental data on the electrical and thermal conductivities of CNT composites [12]. Therefore, the electrical conductivity could have similar behavior as that discussed above, since the conductivity exponent in Eq. (2) satisfies the condition: $1<t(\alpha)<2$. That is, the electrical conductivity slowly increases with CNT 
loading; see the inset of Fig. 3. Here, it is interesting to note that a steep increase can be also seen if we re-plot the data using a semi-logarithmic scale for electrical conductivity, i.e., $\log \sigma$ versus CNT loading, $\left(\phi-\phi_{c}\right)$, indicative of the existence of a percolation threshold [14]. Similarly, we can consider the conductivity in thermal transport measurements by Biercuk et al. [15], which was cited as evidence that there was a lack of thermal percolation in CNT composites [16]. It is seen that, as shown in Fig. 3, the available experimental data of both electrical and thermal conductivities show a slow rise near the percolation threshold although the data of thermal conductivity are too few to see a steep increase, similar to electrical conductivity, in the rescaled plot of $\log \sigma$ versus $\left(\phi-\phi_{c}\right)$. Thus, similar to other physical properties such as electrical conductivity and permeability, a thermal percolation may exist in CNT composites [17]. As a matter of fact, the distinction between these two transport behaviors can be easily explained by percolation theory: the thermal resistance is fully determined by the percolation cluster of CNTs, but the electrical resistance is governed only by the backbone of percolation cluster.

In conclusion, anomalous electrical behavior of CNT composites and suspensions with ultra-low loading of fillers is studied by percolation theory. With the help of the concept of renormalization, a simple model is proposed to estimate the electrical percolation threshold of CNTs in polymer matrix. The results show that the percolation threshold of CNTs is inversely proportional to their aspect ratios. To decrease the percolation threshold of expensive CNTs, there are two efficient ways: one is to use long CNTs and the other is to increase the strength of interaction between them. Near the percolation threshold, the difference between electrical and thermal transport phenomena can be well described by percolation theory. Finally, it is worth noting that the model developed here could be easily extended to the study of other amazing physical properties of CNT-composite films such as optical transparency. 
Acknowledgements This work was supported by the Curtin Internal Research Grants and the Australian Research Council.

\section{References}

1. Zallen R (1983) The Physics of Amorphous Solids, John Wiley \& Sons, Inc., New York

2. Stauffer D, Aharony A (1994) Introduction to Percolation Theory, Taylor \& Francis, London

3. Sandler JKW, Kirk JE, Kinloch IA, Shaffer MSP, Windle AH (2003) Polymer 44:5893

4. Bryning MB, Islam MF, Kikkawa JM, Yodh AG (2005) Adv Mater 17:1186

5. Vigolo B, Coulon C, Maugey M, Zakri C, Poulin P (2005) Science 309:920

6. Stankovich S, Dikin DA, Dommett GHB, Kohlhaas KM, Zimney EJ, Stach EA, Piner RD, Nguyen ST, Ruoff RS (2006) Nature 442:282

7. Kovacs JZ, Velagala BS, Schulte K, Bauhofer W (2007) Compos Sci Technol 67:922

8. Choi SUS, Zhang ZG, Yu W, Lockwood FE, Grulke EA (2001) Appl Phys Lett 79:2252

9. Lu C, Mai Y-W (2005) Phys Rev Lett 95:088303

10. Lu C, Mai Y-M (2007) Compos Sci Technol 67:2895

11. Takayasu H (1990) Fractals in the Physical Sciences, Manchester University Press, Manchester

12. Garboczi EJ, Snyder KA, Douglas JF, Thorpe MF (1995) Phys Rev E 52:819

13. Foygel M, Morris RD, Anez D, French S, Sobolev VL (2005) Phys. Rev. B 71:104201

14. Du F, Fischer JE, Winey KI (2005) Phys Rev B 72:121404

15. Biercuk MJ, Liaguno MC, Radosavljevic M, Hyun JK, Johnson AT, Fischer JE (2002) Appl Phys Lett 80:2767 
16. Shenogina N, Shenogin S, Xue L, Keblinski P (2005) Appl Phys Lett 87:133106

17. Gojny FH, Wichmann MHG, Fiedler B, Kinloch IA, Bauhofer W, Windle AH, Schulte K (2006) Polymer 47:2036 


\section{Figure captions}

Fig. 1 Electrical (or thermal) conductivity of CNT composites versus loading of CNTs, where $\sigma_{0}$ is reference conductivity and the measured percolation thresholds, $\phi_{c}$, are of the order of 0.1 vol. $\%$ or less. The dashed curve shows the percolation probability.

Fig. 2 Illustration of the renormalization model, where there are 7 different topological configurations labelled $0,1,2 \mathrm{a}, \ldots, 4$ with their multiplicities in parentheses. Of total $2^{4}=16$ combinations, there are 5 cases in which CNTs form a conductive path (highlighted in bold letters underneath relevant configurations).

Fig. 3 Electrical conductivity of single walled CNT/poly(methyl methacrylate) (PMMA) composites and thermal enhancement of single walled CNT/epoxy composites as functions of CNT loading $\left(\phi-\phi_{c}\right)$, with $\phi_{c}=0.365$ wt. $\%$ for CNT/PMMA composites and $\phi_{c}=0.15$ wt. $\%$ for CNT/epoxy composites $[14,15]$. 


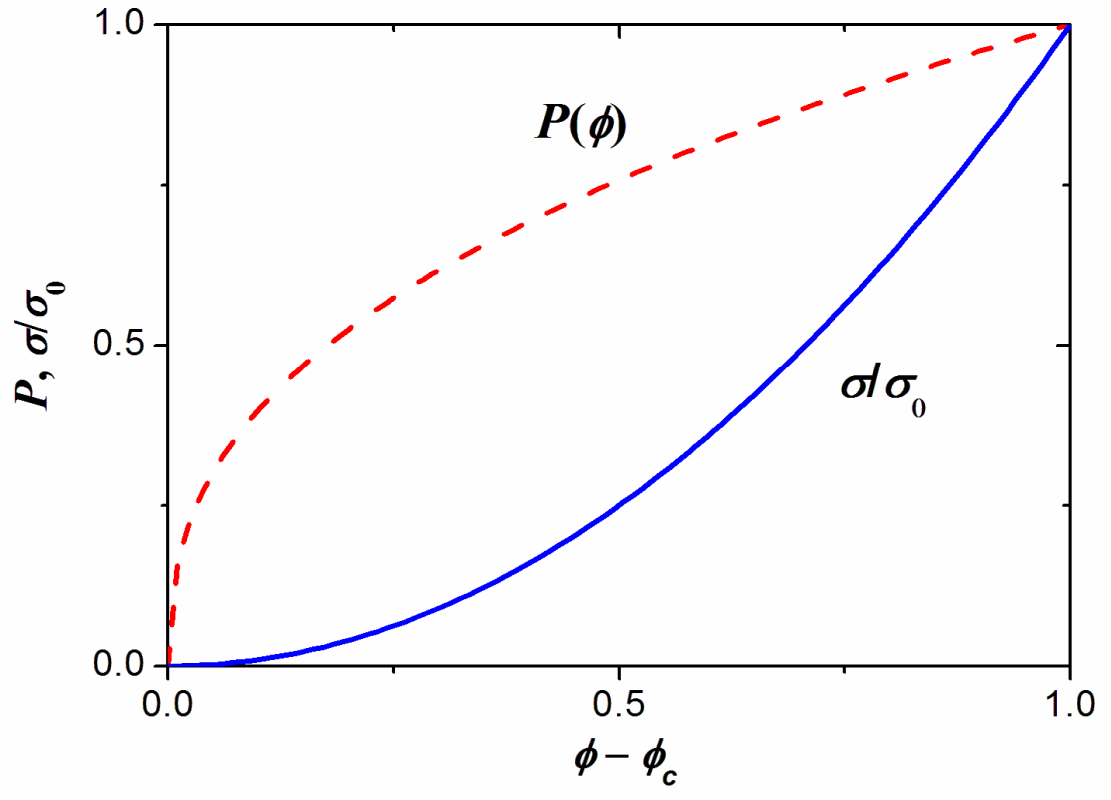

Fig. 1 


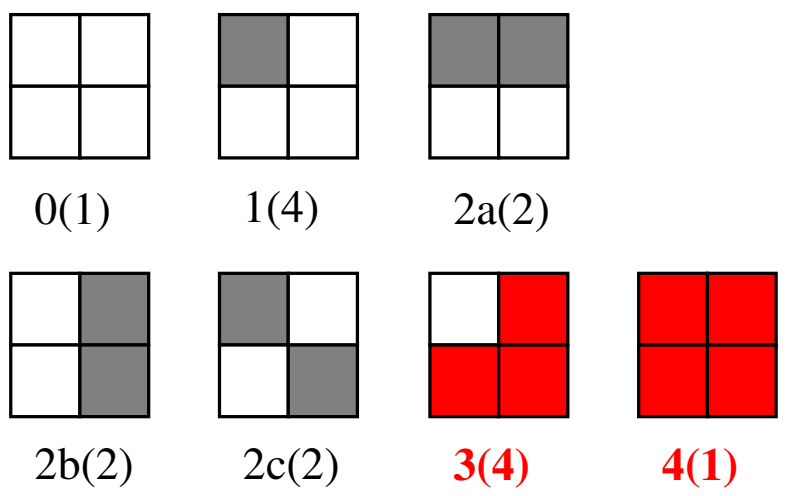

Fig. 2 


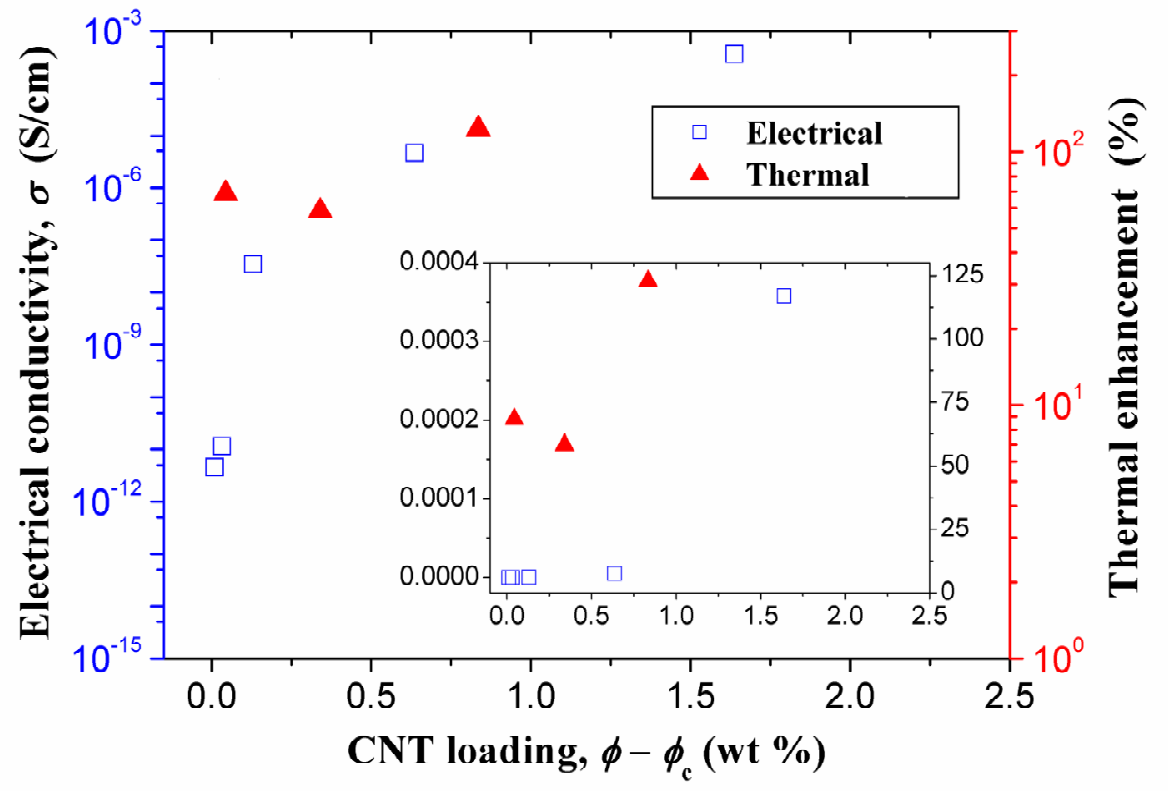

Fig. 3 